\title{
APLICAÇÃO DA TÉCNICA DE CAMINHAMENTO ELÉTRICO EM ÁREA CONTAMINADA POR DERIVADOS DE PETRÓLEO
}

\author{
César Augusto Moreira ${ }^{1}$, João Carlos Dourado² e Antonio Celso de Oliveira Braga ${ }^{2,3}$ \\ Recebido em 3 abril, 2006 / Aceito em 5 setembro, 2006 \\ Received on April 3, 2006 / Accepted on September 5, 2006
}

\begin{abstract}
Geophysical methods are useful technics of geological investigation, thoroughly employed to diagnosis and monitoring contaminated areas, in conjunction with direct techniques of investigation such as chemical analyses. Among these, electric resistivity is more usually used in studies of contaminants in soil and groundwater, due to the high contrast of electric properties between the soil and the pollutant types frequently found, essentially constituted of composed organic and inorganic. Geophysical studies in impacted areas by petroleum products may be describe by anomalies of both low resistivity and high resistivities, confirmed as contaminant by chemical analyses. This apparent contradiction can reflect processes of degradation of the contaminants, directly associated with its residence time in the soil, through the generation of by-products that change the physical properties of the soil and groundwater, principally for the mineral dissolution by action of organic acids and by formation of minerals of oxides and hydroxides minerals. Natural attenuation defines a series of physical, chemical and biological processes that allow the degradation, dispersion and dilution of contaminants in a natural form, in other words, free from human intervention. This paper presents the application of electrical profiling technique in a contaminated industrial area for benzene, toluene, xylene, 1,2 dichloroethene and inorganic salts and it discusses the physical alterations of the contaminated soil through the obtained results, under the optics of the Natural Attenuation in course in the area of study.
\end{abstract}

Keywords: Electrical profiling, electric resistivity, natural attenuation, biodegradation, NAPL.

RESUMO. Os métodos geofísicos são ferramentas consagradas de investigação do meio físico, amplamente empregadas no diagnóstico e monitoramento de áreas contaminadas, sempre em conjunto com técnicas diretas de investigação, como análises químicas. Dentre estes, a eletrorresistividade é uma das ferramentas mais comumente utilizadas em estudos de contaminantes em solo e água subterrânea, devido ao elevado contraste de propriedades elétricas entre o meio geológico e os tipos contaminantes freqüentemente encontrados neste meio, essencialmente constituídos por compostos orgânicos e inorgânicos. Estudos geofísicos em áreas impactadas por derivados de petróleo descrevem anomalias tanto de baixa resistividade quanto de elevada resistividade, confirmadas como fase contaminante por meio de análises químicas. Este aparente contradição pode refletir processos de degradação, associado ao tempo de residência de contaminantes no meio, por meio da geração de subprodutos que alteram as propriedades físicas do meio, principalmente pela dissolução mineral por ação de ácidos orgânicos e pela neoformação de minerais de óxidos e hidróxidos de ferro. Atenuação Natural define uma série de processos físicos, químicos e biológicos que permitem a degradação, dispersão e diluição de contaminantes de forma natural, ou seja, livre da intervenção humana. Este trabalho apresenta a aplicação da técnica de caminhamento elétrico em uma área industrial contaminada por benzeno, tolueno, xileno, 1,2 dicloroetano e sais inorgânicos e discute as alterações físicas do meio contaminado por meio dos resultados obtidos, sob a ótica dos processos de atenuação natural em curso na área de estudos.

Palavras-chave: Caminhamento elétrico, resistividade elétrica, atenuação natural, biodegradação, NAPL.

\footnotetext{
1Pós-graduação em Geociências em Meio Ambiente, IGCE - UNESP, Campus de Rio Claro, Rua 10, no. 2527 - Bairro Santana, 13500-230 Rio Claro, SP, Brasil. E-mail: cesargeologia@yahoo.com.br

2 Departamento de Geologia Aplicada, IGCE - UNESP, Campus de Rio Claro, Av. 24-A no. 1515 - Bela Vista, Caixa Postal 178, 13506-900 Rio Claro, SP, Brasil. E-mail: jdourado@rc.unesp.br

3E-mail: acobraga@rc.unesp.br
} 


\section{INTRODUÇÃ̃o}

0 intenso processo de industrialização iniciado a partir da segunda metade do século XX propiciou o crescimento econômico do Estado de São Paulo. Este crescimento resultou na geração de empregos e renda, elevando o padrão e a qualidade de vida de seus habitantes.

0 resultado negativo deste processo é 0 extenso passivo ambiental herdado de décadas de falta de planejamento e descaso com o meio ambiente, com a degradação de rios, solo e água subterrânea. 0 órgão ambiental estadual havia registrado, até maio de 2002, um total de 255 áreas contaminadas (CETESB, 2001). 0 diagnóstico e o monitoramento de áreas potencialmente contaminadas são necessários devido ao risco à saúde e a qualidade de vida da população.

Os métodos geofísicos são uma alternativa no diagnóstico de áreas contaminadas, devido à rapidez e 0 baixo custo em estudos ambientais. Esta ferramenta pode auxiliar na detecção e no monitoramento de áreas contaminadas, em conjunto com métodos diretos de investigação, como poços de monitoramento. As técnicas geofísicas de Sondagem Elétrica Vertical (SEV), Radar de Penetração no Solo (GPR) e Eletromagnético Indutivo (EM) tem sido cada vez mais aplicadas para a caracterização e monitoramento de áreas impactadas por contaminantes por Fase Líquida Não Aquosa (NAPL) (Atekwana et al., 2000; Readman et al., 1994; Ellert et al., 1988).

Diversos trabalhos descrevem a aplicação dos métodos geofísicos combinados com análises diretas de investigação do meio impactado, na caracterização de contaminantes derivados de petróleo em diversas áreas.

A aplicação combinada da técnica de Caminhamento Elétrico e Sondagem Elétrica Vertical em Lima et al. (1995), descreve a detecção de plumas de contaminação provenientes de um aterro de resíduos líquidos derivados de petróleo, cuja correlação entre pseudo-seções de resistividade aparente e um número muito reduzido de poços de monitoramento permitiu associar intervalos de baixa resistividade com áreas de concentração de contaminantes.

Estudos de vazamentos de compostos de fase líquida leve não aquosa (LNAPL) por meio de perfis GPR por Bermejo et al. (1997), permitiram a correlação entre reflexões tipo zona de sombra com compostos de elevada condutividade localizados na parte superior do aqüífero, com valores medidos em amostras de solo e água de 2,5 a 3,3 vezes superiores ao padrão da área.

Os trabalhos de Benson \& Stubben (1995) e Sauck (1998) relacionam a presença de zonas de mancha em perfis GPR com contaminações antigas de LNAPL, caracterizadas por anomalias de baixa resistividade em relação padrão natural do aquífero.

Em contrapartida, o uso combinado de métodos elétricos e eletromagnéticos por Benson et al. (1997) no mapeamento de uma pluma de contaminação composta por benzeno, tolueno, xileno e etil-benzeno (BTEX) e com elevada quantidade de sólidos totais dissolvidos, resultou na detecção de uma anomalia de alta resistividade, associada a elevadas concentrações de hidrocarbonetos sob a forma de fase livre pouco degradada, predominante em relação à quantidade de sólidos totais dissolvidos. As zonas de mancha por reflexões de onda em perfis GPR apresentadas por Benson (1995), são interpretadas como hidrocarbonetos provenientes de vazamentos em tanques enterrados, contaminantes caracterizados por resistividade superior ao padrão do aqüífero. A presença de fase livre sobrenadante foi verificada posteriormente em poços de monitoramento.

As respostas geofísicas em meios impactados por contaminantes derivados de hidrocarbonetos apresentam anomalias tanto de elevada resistividade como de baixa resistividade. Esta aparente incongruência pode ser esclarecida por meio da correlação do tempo de residência dos contaminantes no solo e tempo de exposição a processos de degradação. Alguns trabalhos sugerem que a biodegradação de compostos (LNAPL) pode mudar as propriedades geoquímicas do aqǘfero de forma acentuada, que podem chegar a alterações significativas em medidas de resistividade e na propagação de ondas eletromagnéticas (Sauck, 2000; Atekwana et al., 2000). Estudos em escala de laboratório sugerem que a biodegradação de LNAPL sob condições aeróbicas e anaeróbicas podem mudar de forma substancial as condições biogeoquímicas do aqüifero impactado, resultando em reduções drásticas de resistividade elétrica (Cassidy et al., 2001).

A degradação de contaminantes por ação de microorganismos, denominada biodegradação, é um dos principais mecanismos responsáveis pela degradação de contaminantes com longos períodos de residência no ambiente (EPA, 1999). A eficiência deste processo depende de uma conjunção de fatores, como a presença de receptores elétricos $\left(\mathrm{O}_{2}, \mathrm{SO}_{4}^{2-}, \mathrm{Fe}_{3}^{+}, \mathrm{Mn}_{4}^{+}, \mathrm{NO}_{3}^{-}\right)$, condições adequadas de $\mathrm{pH}$, Eh, temperatura e baixos teores de contaminação (EPA, 1998).

Este trabalho apresenta a aplicação da técnica de caminhamento elétrico em uma área industrial contaminada por benzeno, tolueno, xileno, 1,2 dicloroetano e sais inorgânicos e discute as alterações físicas do meio contaminado a partir dos resultados obtidos, sob a ótica do processo de biodegradação de compostos de fase líquida não aquosa (NAPL). 


\section{MATERIAIS E MÉTODOS Atenuação Natural \\ Definição}

A Atenuação Natural compreende um trabalho de remediação que inclui uma variedade de processos físicos, químicos e biológicos que, dentro de condições favoráveis, permite a redução de massa, toxicidade, mobilidade, volume ou concentrações de contaminantes no solo ou água subterrânea sem intervenção humana. Este processo ocorre naturalmente em qualquer área contaminada, com variável grau de eficiência, dependendo dos tipos e concentrações de contaminantes presentes e dos processos físicos, químicos e biológicos característicos do solo e da água subterrânea (EPA, 2004).

Este processo é importante para a degradação de derivados de petróleo, que podem ser degradados por organismos encontrados em quase todos os ambientes. Vários compostos de fase líquida leve não aquosa (LNAPL), entre eles os BTEX (benzeno, tolueno, etilbenzeno e xileno) podem ser biodegradados sob condições ambientais (EPA, 1998).

Contudo, este processo natural pode ser limitado pela falta de nutrientes, elétrons receptores ou ambos (Thomas \& Ward, 1989). Em muitos locais, o processo de biodegradação fica limitado as zonas periféricas da pluma contaminante, pois a presença de contaminante em elevadas concentrações torna 0 ambiente extremamente hostil a proliferação de microorganismos (Wilson \& Jones, 1993; Rabus \& Heider, 1998).

\section{Consequiências ao Meio Contaminado}

A degradação de derivados de petróleo por metabolismo microbial produz uma variedade de subprodutos reativos, muitos dos quais interagem diretamente com solutos inorgânicos dissolvidos e minerais presentes. Estes subprodutos podem acelerar reações de dissolução e precipitação, complexação de íons metálicos em solução, alterações no estado de valência de íons metálicos e mobilização de metais solúveis (Harter, 1977).

Poucos trabalhos abordam a dissolução mineral por ação de ácidos orgânicos e sistemas aqǘferos rasos, provenientes da degradação de contaminantes derivados de petróleo (McMahon et al., 1995; Hiebert \& Bennett, 1992).

A dissolução mineral pode desencadear processos como:

- Geração de porosidade intragranular, que inibe a recuperação de íons metálicos e derivados de petróleo em aquíferos de elevada porosidade (Stoessel \& Pittman, 1990; Barth \& Riis, 1992).
- Dissolução de óxidos de ferro, que pode resultar na liberação de íons metálicos em determinados ambientes (McCabe et al., 1987).

- Neoformação mineral, com fechamento de espaços porosos previamente estabelecidos e modificação no padrão de circulação da água subterrânea, que dificultam os esforços de remediação.

\section{LOCALIZAÇÃO E HISTÓRICO DA ÁREA}

A área de estudos está localizada no município de Araras, estado de São Paulo, precisamente no Km 51,2 da rodovia SP 191, que liga as cidades de Araras e Rio Claro (Figura 2). 0 acesso a partir da rodovia SP 330 é feito no cruzamento com a rodovia SP 191, a partir do qual o local de estudos está distante cerca de $1400 \mathrm{~m}$ sentido Rio Claro.

0 local foi ocupado pela Redistil Indústria Química Ltda e pela Indústria Química Sulfabrás S/A, entre os anos de 1981 e 1992. Dentre os produtos fabricados estão o dieloxalato, acetato de etila, 4-amino-N-2-tiozolil-benzeno-sulfonamida, acetopiruvato de metila, 5-metil-3-carboxiamidoxazol (carboxiamido), e dentre os solventes utilizados durante 0 processo estavam 0 1,2 dicloroetano e o tolueno (CETESB, 1997).

Os solventes utilizados por ambas as indústrias eram reutilizados após passarem por colunas de destilação. As águas residuais deste processo passavam por um tacho para concentrar os efluentes. Em seguida, os efluentes gerados eram infiltrados em dois poços presentes no local. Este processo foi executado entre os anos de 1981 e 1988 (CETESB, 1997).

Em novembro de 1985 foram detectados odores em poços de abastecimento nos arredores da área de infiltração. Análises químicas realizadas em amostras coletadas em poços próximos a indústria indicaram contaminação da água subterrânea (Tabela 1). Posteriormente foram efetuadas análises químicas nos resíduos infiltrados com vistas á caracterização de sua composição química, cujo resultado permitiu correlação com os contaminantes presentes nas águas subterrâneas (Tabela 2) (CETESB, 1997).

A partir de 1992, com o fechamento da indústria, a CETESB iniciou o monitoramento da atenuação natural dos contaminantes, a partir de diversos poços de monitoramento instalados na área e adjacências, em conjunto com a aplicação de técnicas geofísicas (CETESB, 1997; Aquino, 2000). Sucessivas análises químicas realizadas em amostras coletadas no poço de monitoramento localizado na indústria Fuganholi (Pm8) indicam decréscimo no teor de contaminantes ao longo de nove anos (Tabela 3). 


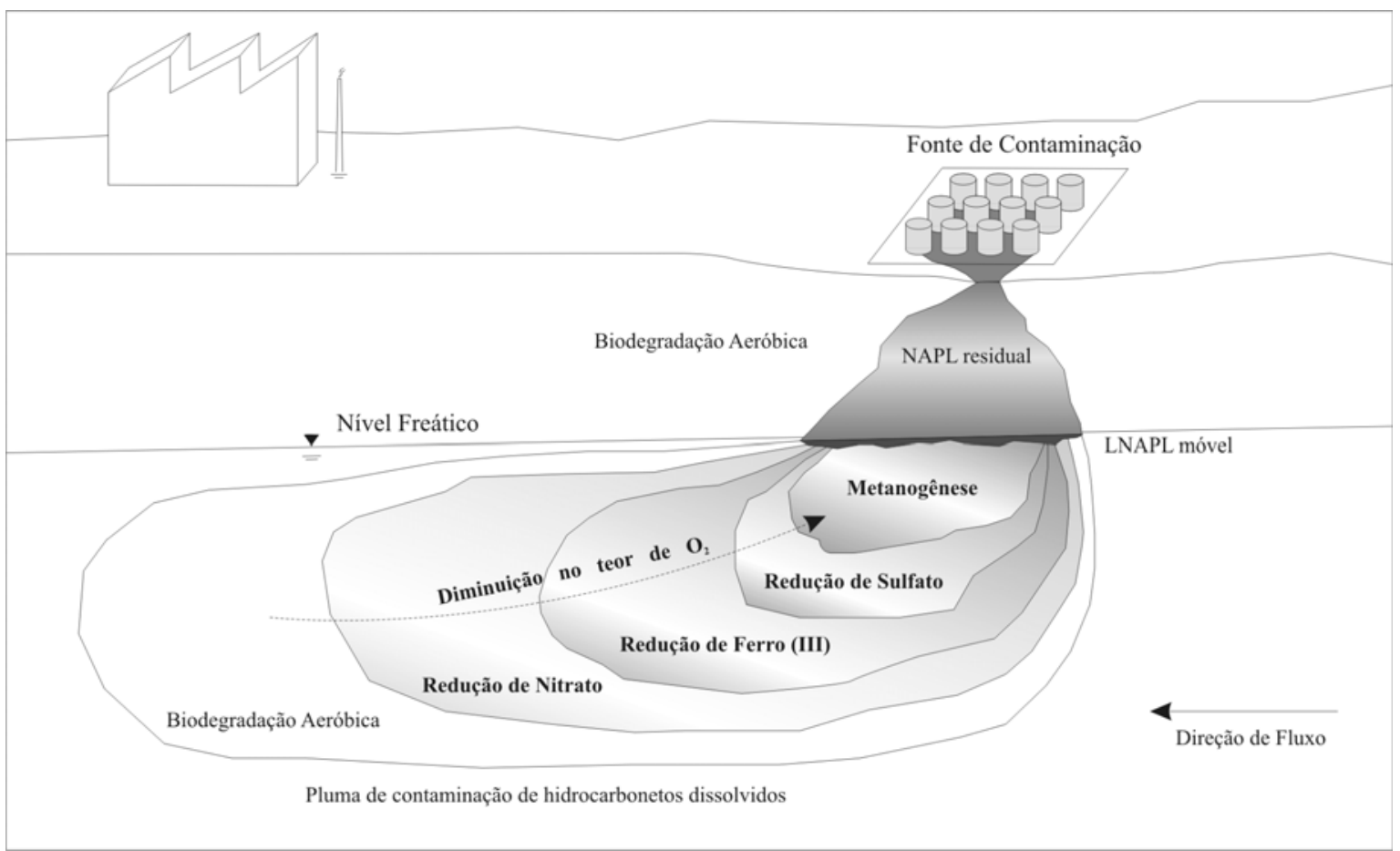

Figura 1 - Zonas de degradação de hidrocarbonetos (adaptado de Rabus \& Heider, 1998).

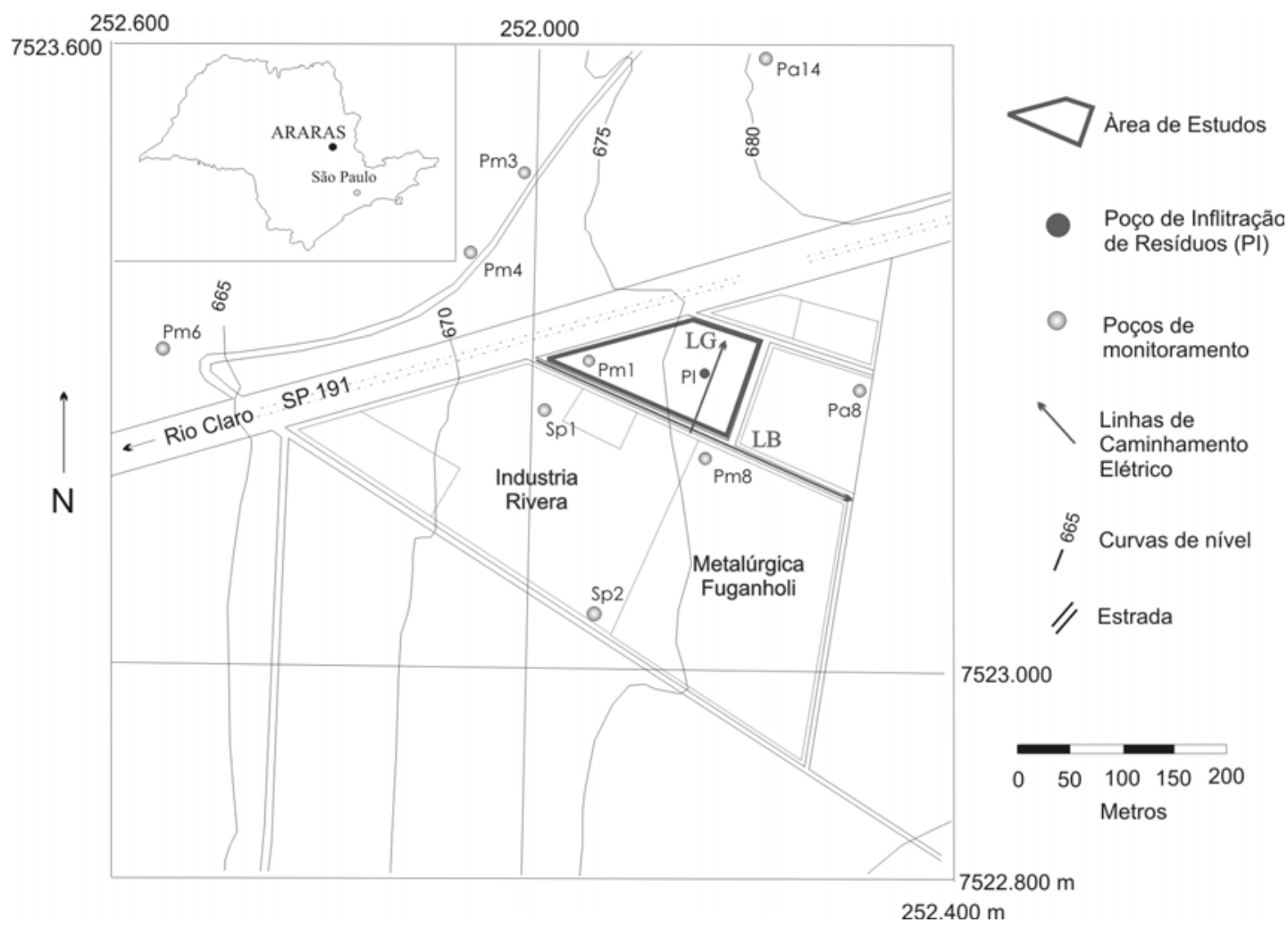

Figura 2 - Mapa de localização com linhas de caminhamento elétrico. 
Tabela 1 - Análises químicas em amostras de água subterrânea coletadas em poços de monitoramento na área da indústria Sulfabrás (1992) (CETESB, 1997).

\begin{tabular}{|c|c|c|c|c|c|}
\hline Poç0 & Benzen0* $^{*}$ & Tolueno* $^{*}$ & Xileno* $^{*}$ & Clorofórmio* $^{*}$ & 1,2 Dicloroetano* \\
\hline PS & ND & ND & ND & ND & ND \\
\hline P1 & 543,0 & 117,0 & 10,0 & ND & ND \\
\hline P2 & ND & $<1,0$ & ND & ND & ND \\
\hline P3 & ND & 31,0 & 21,0 & ND & ND \\
\hline P4 & 146,0 & ND & ND & 1,5 & ND \\
\hline P5 & 77,0 & ND & ND & ND & ND \\
\hline P6 & 33,0 & $<1,0$ & ND & $<1,0$ & $<1,0$ \\
\hline P7 & ND & ND & ND & ND & ND \\
\hline P8 & $6,0 \times 10^{3}$ & $7,0 \times 10^{3}$ & ND & 133,0 & 38,0 \\
\hline VMP & 10 & 1 & 10 & 30 & 10 \\
\hline \hline P0ç0 & Cloreto** & N Amoniacal** & N Nitrato** & Sódio** & Sulfato** \\
\hline PS & $<0,5$ & 0,12 & 0,02 & 6,7 & 2,0 \\
\hline P1 & $1,5 \times 10^{3}$ & 12,0 & 0,03 & 690,0 & 30,0 \\
\hline P2 & $<0,5$ & 0,09 & 0,05 & 2,0 & 26,0 \\
\hline P3 & $<0,5$ & 0,01 & 0,02 & 0,1 & 20,0 \\
\hline P4 & 110,0 & 0,08 & 0,02 & 41,0 & 27,0 \\
\hline P5 & 65,0 & 0,07 & 0,12 & 15,0 & 25,0 \\
\hline P6 & 35,5 & 0,02 & 0,20 & 15,5 & 24,0 \\
\hline P7 & 1,0 & 0,02 & 0,22 & 0,4 & 24,0 \\
\hline P8 & $2,15 \times 10^{3}$ & 240,0 & 0,05 & $1,8 \times 10^{3}$ & 31,0 \\
\hline VMP & 250 & - & 10 & 200 & 400 \\
\hline & & & & & \\
\hline
\end{tabular}

$\mathrm{ND}=$ Não detectado; VMP = Valores máximos permitidos; *valores em $\mu \mathrm{g} / \mathrm{L} ;{ }^{* \star}$ valores em mg/L.

Tabela 2 - Análises químicas em amostra de água coletada no poço de infiltração de resíduos reconhecido na indústria Sulfabrás (1987) (CETESB, 1997).

\begin{tabular}{|c|c|c|c|c|c|}
\hline 1,2 Dicloroetano* & Benzeno* $^{*}$ & Tolueno $^{*}$ & Xileno* $^{*}$ & Cloreto** $^{*}$ & $\mathrm{pH}$ \\
\hline $7,5 \times 10^{4}$ & $\mathrm{ND}$ & $1,7 \times 10^{4}$ & $\mathrm{ND}$ & $1,3 \times 10^{7}$ & 9,6 \\
\hline
\end{tabular}

$\mathrm{ND}=$ Não detectado; * valores em $\mu \mathrm{g} / \mathrm{L} ;{ }^{* *}$ valores em mg/L.

Tabela 3 - Análises químicas em amostras coletadas no poço de monitoramento (Pm8) localizado na metalúrgica Fuganholi (CETESB, 1997).

\begin{tabular}{|l|c|c|c|}
\hline Contaminante & $06 / 1990$ & $01 / 1992$ & $11 / 1999$ \\
\hline Benzeno $(\mu \mathrm{g} / \mathrm{L})$ & $29,5 \times 10^{3}$ & $6,0 \times 10^{3}$ & $1,19 \times 10^{4}$ \\
\hline Tolueno $(\mu \mathrm{g} / \mathrm{L})$ & $24,5 \times 10^{3}$ & $7,0 \times 10^{3}$ & $1,39 \times 10^{4}$ \\
\hline 1,2 Dicloroetano $(\mu \mathrm{g} / \mathrm{L})$ & $12 \times 10^{3}$ & 36,0 & 15,9 \\
\hline Sódio $(\mathrm{mg} / \mathrm{L})$ & 200,0 & $2,1 \times 10^{3}$ & - \\
\hline Cloreto $(\mathrm{mg} / \mathrm{L})$ & $2,8 \times 10^{3}$ & $1,8 \times 10^{3}$ & $1,35 \times 10^{3}$ \\
\hline Sulfato $(\mathrm{mg} / \mathrm{L})$ & 32 & 31 & $<10$ \\
\hline
\end{tabular}




\section{Fisiografia}

A área está localizada na porção nordeste da bacia sedimentar do Paraná, composta por rochas Paleozóicas (Grupo Itararé, Formações Tatuí, Irati e Corumbataí), Mesozóicas (Formação Pirambóia) e Cenozóicas (Formação Rio Claro e depósitos atuais) constituídas por sedimentos, derrames e intrusões básicas (CPRM, 1986).

0 meio geológico local é caracterizado por siltitos argilosos pertencentes à Formação Tatuí, alterados em superfície para solo vermelho escuro argiloso, com matriz de fração silte entre 1 e $3 \mathrm{~m}$ de profundidade e matriz de fração argila entre 3 a $13 \mathrm{~m}$ de profundidade, com transição gradual entre os níveis (Figura 3). 0 nível freático está situado entre 10,7 e 14,1 m de profundidade, com oscilações máximas entre 7,9 e 14,9 m. 0 fluxo da água subterrânea nas proximidades dos pontos de infiltração de resíduos é para sudoeste. A condutividade hidráulica obtida em ensaios nos poços de monitoramento varia de $4,4 \times 10^{-7}$ a 7, $0 \times 10^{-7} \mathrm{~cm} / \mathrm{s}$ (CETESB, 1997; Aquino, 2000).

\section{CAMINHAMENTO ELÉTRICO}

A técnica de caminhamento elétrico está baseada na realização de medidas de resistividade aparente ao longo de uma linha, com 0 objetivo de investigar variações em um ou mais níveis em profundidade (Sherrif, 1989). Dentre os diversos tipos de arranjos de campo disponíveis para a técnica do caminhamento elétrico, o presente trabalho utiliza o arranjo dipolo-dipolo (Figura 4).

0 arranjo dipolo-dipolo é caracterizado por utilizar espaçamento igual entre $M N$ e $A B$, com deslocamento do centro de ambos os dipolos ao longo da linha. 0 espaçamento entre os dipolos $A B$ e MN pode ser variado a partir do uso simultâneo de vários dipolos MN ao longo da linha.

Este trabalho apresenta três linhas de caminhamento elétrico (Figura 2). A linha LG apresenta comprimento total de 85 metros e a linha LB apresenta $340 \mathrm{~m}$ de comprimento, realizadas em arranjo dipolo-dipolo com espaçamento de $10 \mathrm{~m}$ entre eletrodos e leituras em 5 níveis de profundidade. Em ambos os perfis, as cores quentes (laranja-violeta) indicam elevada resistividade, enquanto que as cores frias (verde-azul) indicam baixa resistividade.

\section{RESULTADOS}

A profundidade média de 8,5 m define o contato entre a zona nãosaturada e a zona saturada do aqüífero, com nível freático posicionado entre torno de 12 m, definido por meio de sondagens á percussão, realizadas nas adjacências das áreas (CETESB, 1997).
A anomalia de alta resistividade associada ao ponto de injeção de resíduos - forma triangular na posição 52,5m na linha LG (Figura 4), é resultado da presença de elevadas concentrações de compostos contaminantes. Estes compostos possivelmente estão em fase residual - aderidos aos grãos minerais do solo, visto que as análises químicas indicam redução dos teores de contaminantes em água (Tabela 3). Este intervalo deve corresponder à zona de metanogênese (Figura 1), caracterizada por elevadas concentrações de contaminantes e alta toxicidade para ação efetiva dos processos de degradação por microorganismos. Neste contexto, os compostos deste intervalo estão relativamente mais preservados e com características físicas menos alteradas, possivelmente devido à oxigenação pouco efetiva associada à condutividade hidráulica bastante reduzida da área.

A partir de experimentos em escala reduzida, Braga \& Cardinalli (2005) demonstram 0 aumento da resistividade elétrica em sedimentos arenosos após a adição de gasolina. Durante 0 intervalo de seis meses houve gradativa redução da resistividade, que culminaram com valores mais condutivos do que meio não contaminado.

As anomalias de baixa resistividade presentes na porção inicial da linha LG e posição centro-direita na linha LB (Figura 5), representam um único corpo anômalo, pois o intervalo condutivo da linha LB corresponde a uma continuação da anomalia descrita no início da linha LG, que por sua vez está situada a montante do fluxo d'água subterrânea (Figura 5).

Os compostos orgânicos são divididos em fase residual e fase dissolvida quando injetados no meio geológico (Rabus \& Heider, 1998). A fase dissolvida apresenta alta mobilidade, pois migra quase concomitantemente ao fluxo da água subterrânea, enquanto que a fase residual representa o contaminante que impregna os grãos minerais.

Uma vez atingida a solubilidade máxima em água, a fase dissolvida tende a se dispersar e a reduzir seus teores. A partir deste momento, são criadas condições favoráveis para uma ação efetiva do processo de degradação por microorganismos, devido à redução da toxicidade e aumento no teor de oxigênio (Figura 1).

A ação de microorganismos resulta na geração de ácidos orgânicos, que em quantidade elevadas passam a atacar os grãos minerais que constituem o meio (Ehrlich, 1996). Este processo resulta na dissolução mineral e liberação de íons para a zona saturada do aqüífero, com conseqüente alteração nos padrões de resistividade elétrica em relação ao meio não contaminado (McMahon \& Chapelle, 1991). Este comportamento pode ser observado essencialmente na posição centro-direita da linha LB e na porção inicial na linha LG. 


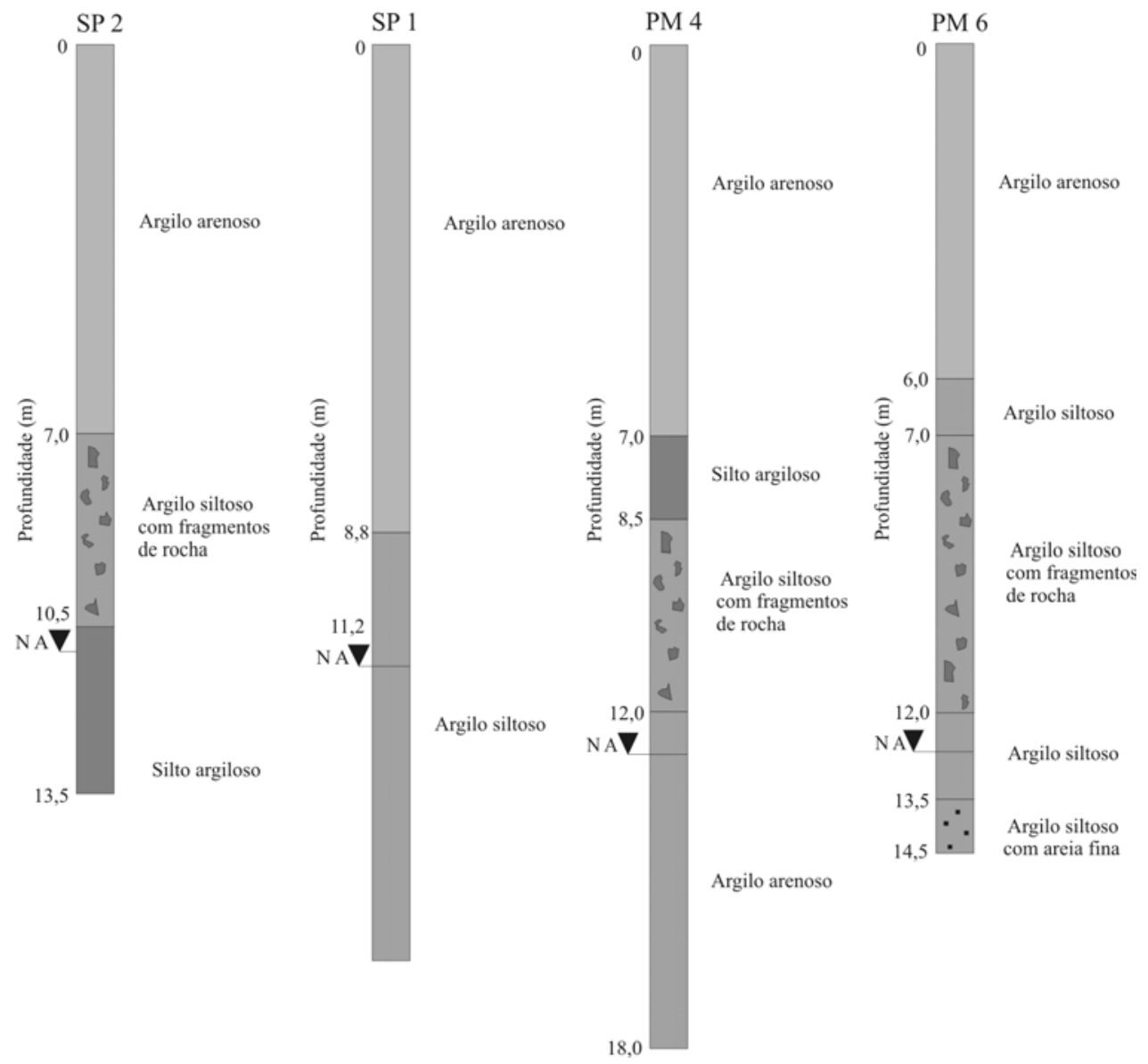

Figura 3 - Descrições de sondagem com profundidade em metros (CETESB, 1997).

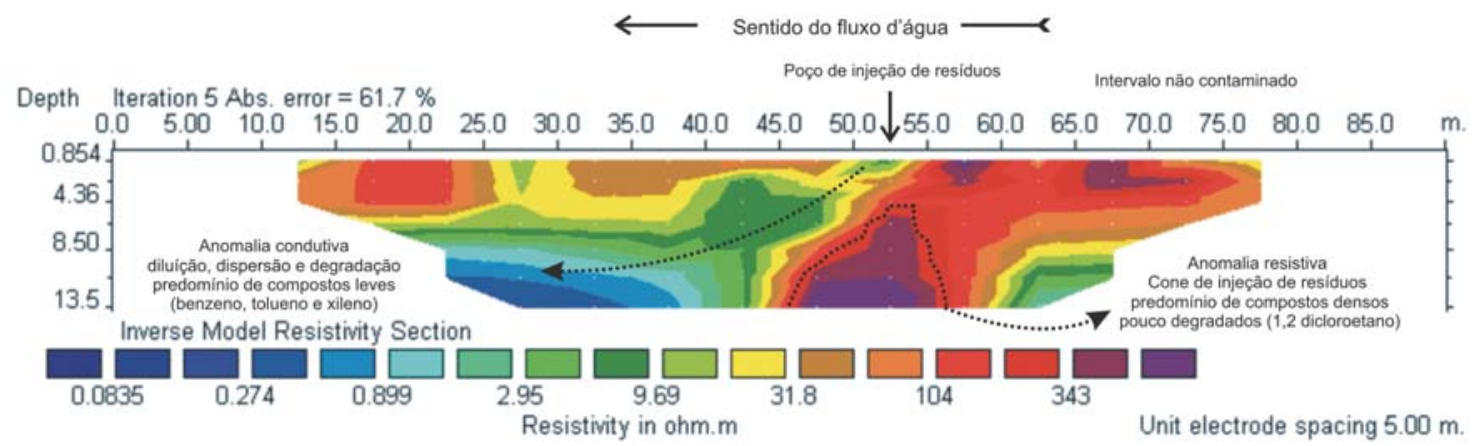

Figura 4 - Modelo de inversão da linha LG.

0 erro percentual apresentado no modelo de inversão e resultado do contraste bastante acentuado nos valores de resistividade entre as porções provavelmente contaminadas e o meio em redor. Apesar deste fato, os modelos de inversão estão condizentes com trabalhos geofísicos anteriores realizados na área (CETESB, 1997; Aquino, 2000; Moreira \& Dourado, 2003 e 2005).

\section{CONCLUSÕES}

0 corpo de baixa resistividade é possivelmente resultado de mudanças nas propriedades físicas do meio contaminado. A causa principal é a presença de íons dispersos principalmente na zona saturada do perfil de alteração, em quantidades superiores aos que ocorrem naturalmente na área, cujo contraste permi- 


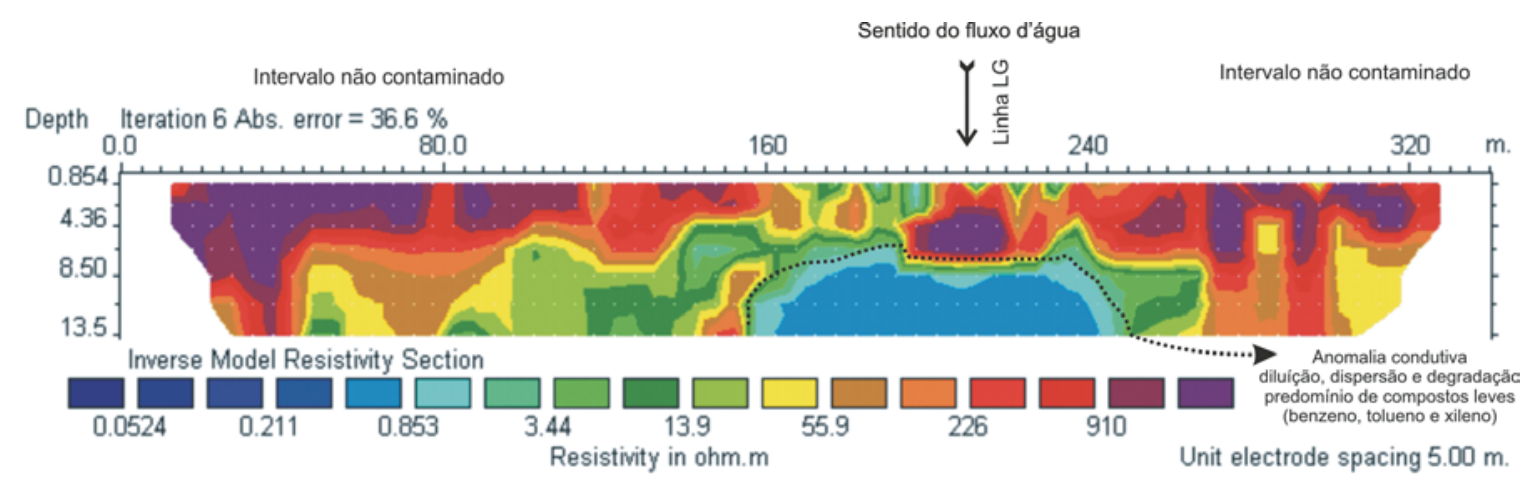

Figura 5 - Modelo de inversão da linha LB.

tiu sua determinação a partir do parâmetro resistividade elétrica (Figura 4 e 5). Estes íons são produto da corrosão de grãos minerais que compõem o meio, por ataque de ácidos orgânicos.

A anomalia de alta resistividade praticamente restrita a porção inferior do poço de injeção de resíduos, pode indicar elevados teores de contaminação, predominantemente compostos de fase densa não aquosa (DNAPL) em fase residual, pois a alta toxicidade não permite degradação por ataque de microorganismo. A caracterização da anomalia de alta resistividade foi possível devido posição do ponto de injeção de resíduos, formato cônico da anomalia e pela presença de anomalias de baixa resistividade em redor, pois o contraste de resistividade com o meio não contaminado é pequeno (Figura 5).

A porção resistiva no final da linha LG representa o meio praticamente ausente de contaminação por estar situado a montante do poço de injeção de resíduos. Os extremos da linha LB também representam áreas praticamente ausentes de contaminação, embora neste caso a qualidade desta seção não colabore para tal definição, resultado da presença uma linha de transmissão de energia elétrica paralela a esta seção, aliado ao elevado contraste de valores de resistividade obtidos.

As diferenças nos padrões de resistividade descritos em trabalhos de aplicação do método de eletrorresistividade em áreas contaminadas por derivados de petróleo, podem estar associadas, entre outros fatores, ao tipo de contaminante - fase leve (LNAPL) ou fase densa (DNAPL), a permeabilidade do aqüífero e conseqüente tempo de residência dos contaminantes. Aqüíferos porosos e permeáveis permitem a diluição e dispersão de contaminantes de forma relativamente rápida e eficiente, permitindo oxigenação e criando condições para degradação tanto aeróbica quanto anaeróbica. Aqüíferos pouco permeáveis, como no caso estudado neste trabalho, a mobilidade dos compostos é comprometida, principalmente de fase densa, que resulta em lon- gos períodos de dispersão e diluição, com possibilidade de degradação efetiva somente nas bordas do corpo contaminante.

\section{AGRADECIMENTOS}

0 presente trabalho foi realizado com o apoio do Conselho Nacional de Desenvolvimento Científico Tecnológico - CNPq - Brasil. Os autores agradecem ao técnico Francisco Manuel Garcia Barrera, do Departamento de Geologia Aplicada, UNESP - Rio Claro, pelo auxílio na execução dos trabalhos de campo.

\section{REFERÊNCIAS}

AQUINO WF. 2000. Métodos Geofísicos Eletromagnéticos aplicados ao diagnóstico da contaminação de Solo e das Águas Subterrâneas em área de Infiltração de Resíduos Industriais. Dissertação (Mestrado em Geociências). Instituto de Geociências. Universidade de São Paulo. $121 \mathrm{f}$.

ATEKWANA EA, SAUCK WA, WERKEMA DD. 2000. Investigations of geoelectrical signature at a hydrocarbon contaminated site. Journal of Applied Geophysics, 44: 167-180.

BARTH T \& RIIS M. 1992. Interactions between organic acid anions in formation waters and reservoir mineral phases. Organic Geochemistry, 19: $455-482$.

BENSON AK. 1995. Applications of ground penetrating radar in assessing some geological hazards: examples of groundwater contamination, faults, cavities. Journal of Applied Geophysics, 33: 177-193.

BENSON AK, PAYNE KL \& STUBBEN MA. 1997. Mapping groundwater contamination using dc resistivity and VLF geophysical methods A case study. Geophysics, 62(1): 80-86.

BENSON AK \& STUBBEN MA. 1995. Interval resistivities and very low frequency electromagnetic induction - an aid to detecting groundwater contamination in space and time: A case study. Environmental Geosciences, 2: 74-84. 
BERMEJO JL, SAUCK WA \& ATEKWANA EA. 1997. Geophysical discovery of a new LNAPL plume at the former Wurtsmith AFB. Ground Water Monitoring Remediation, 17(4): 131-137.

BRAGA ACO \& CARDINALI MT. 2005. Aplicação da resistividade e cargabilidade em estudos de contaminação de sedimentos por derivados de Hidrocarbonetos. Revista Brasileira de Geofísica, 23(2): 181-190.

CASSIDY DP, WERKEMA DD Jr., SAUCK W, ATEKWANA E, ROSSBACH $S \&$ DURIS J. 2001. The Effects of LNAPL Biodegradation Products on Electrical Conductivity Measurements. Journal of Environmental \& Engineering Geophysics, 6(1): 47-52.

CETESB. COMPANHIA DE TECNOLOGIA DE SANEAMENTO AMBIENTAL. 1997. Relatório do levantamento geofísico para diagnóstico da contaminação da indústria Sulfabrás, São Paulo: Cetesb, 91 p.

CETESB. COMPANHIA DE TECNOLOGIA DE SANEAMENTO AMBIENTAL. 2001. Relatório de qualidade das águas subterrâneas no Estado de São Paulo. São Paulo: CETESB, pp. 28-29.

CPRM. Companhia de Pesquisa de Recursos Minerais. 1986. Mapa geológico do município de araras, escala 1.100.000. In: Projeto borda leste da bacia do Paraná: integração geológica e avaliação econômica. Relatório final, volume ii, anexos.

EHRLICH HL. 1996. How microbes influence mineral growth and dissolution. Chemical Geology, 132: 5-9.

ELLERT N, GREENHOUSE J \& WILLIANS MM. 1988. A geofísica no estudo da poluição da água subterrânea. Revista Ambiente, 2(2): 21-30.

EPA. UNITED STATES ENVIRONMENTAL PROTECTION AGENCY. 1998. Technical Protocol for evaluating natural attenuation of chlorinated solvents in ground water. Washington DC, epa/600/r-98/128.

EPA. UNITED STATES ENVIRONMENTAL PROTECTION AGENCY. 1999. Microbial processes affecting monitored natural attenuation of contaminants in the subsurface. Ada, Oklahoma, epa/540/s-99/001.

EPA. UNITED STATES ENVIRONMENTAL PROTECTION AGENCY. 2004. Use of monitored natural attenuation at Superfund, RCRA corrective action, and Underground Storage Tank sites. Office of Solid Waste and Emergency Response, Directive 9200. p. 4-17, Chapter IX Monitored Natural Attenuation, final version. 2004. Disponível em: <http://www.epa.gov>. Acesso em 14 jun. 2004.

HARTER RD. 1977. Reactions of minerals with organic compounds in soil. Minerals in Soil Environments, Soil Science Society of America. Madison, Ed. Joe. B. Dixon, Chapter 20. 709-739.

HIEBERT FK \& BENNETT PC. 1992. Microbial Control of Silicate Weathering in Organic-Rich Ground Water. Science: American Association for the Advancement of Science, 258: 278-281.
LIMA OAL, SATO HK \& PORSANI MJ. 1995. Imaging industrial contaminant plumes with resistivity techniques. Journal Applied Geophysics, 34: $93-108$.

MCCABE C, SASSEN R \& SAFFER B. 1987. Occurrence of secondary magnetite within biodegraded oil. Geology, 15: 7-10.

McMAHON PB \& CHAPELLE FH. 1991. Microbial production of organic acids in aquitard sediments and its role in aquifer geochemistry. Nature, 349: 233-235.

MCMAHON PB, VROBLESKY DA, BRADLEY PM, CHAPELLE FH \& GULLETT CD. 1995. Evidence for Enhanced Mineral Dissolution in Organic Acid-Rich Shallow Ground Water. Ground Water, 33(2): 207-216.

MOREIRA CA \& DOURADO JC. 2005. Análise de contaminantes de fase líquida não aquosa (NAPLs) por aplicação do método Eletromagnético Indutivo (EM). Revista Brasileira de Geofísica, 23: 213-220.

MOREIRA CA \& DOURADO JC. 2003. Análise Temporal de Pluma de Contaminação por meio de GPR, In: SBGf, 8th International Congress of the Brazilian Geophysical Society and 5th Latin American Geophysical Conference, Rio de Janeiro (RJ), 2003.

RABUS R \& HEIDER J. 1998. Initial reactions of anaerobic metabolism of alkylbenzenes in denitrifying and sulfate-reducing bacteria. Archives of Microbiology, 170: 377-384.

REDMAN JD, DeRYCK SM, ANNAN AP. 1994. Detection of LNAPL pools with GPR: Theoretical modeling and surveys of a controlled spill. In: Proceedings of the Fifth International Conference on Ground Penetration Radar (GPR 94). Kitchener, Ontario, p. 1795-1294.

SAUCK WA. 1998. A conceptual model for the geoelectrical response of LNAPL Plumes in granulated sediments. Proceedings of the Symposium on the Applications of Geophysics to Engineering and Environmental Problems, Chicago, Illinois, 805-817.

SAUCK WA. 2000. A model for the resistivity structure of LNAPL plumes and their environs in sandy sediments. Applied Geophysics, 44: 151165.

SHERIFF ER. 1989. Geophysical methods, Prentice Hall, chapter 9, Electrical and Electromagnetic methods, p. 192-200.

STOESSEL RK \& PITTMAN ED. 1990. Secondary porosity resisted: The chemistry of feldspar dissolution by carboxylic acids and anions. American Association of Petroleum Geology, 74: 1795-1805.

THOMAS JM \& WARD CH. 1989. In situ biorestoration of organic contaminants in the subsurface. Environ. Sci. Technol., 23: 760-766.

WILSON SC \& JONES KC. 1993. Bioremediation of soils contaminated with Polynuclear Aromatic Hydrocarbons (PAHs): a review. Environmental Pollution, 81: 229-249. 


\section{NOTAS SOBRE OS AUTORES}

César Augusto Moreira. Bacharel em Geologia pelo Instituto de Geociência e Ciências Exatas (IGCE) da Universidade Estadual Paulista (UNESP) em 2003. Atualmente é aluno do programa de Pós-Graduação em Geociências e Meio Ambiente do Instituto de Geociências e Ciências Exatas - UNESP - Campus de Rio Claro.

João Carlos Dourado. Bacharel em Geologia pelo Instituto de Geociências da Universidade de São Paulo (USP) em 1977. Doutor em Geociências pela UNESP em 1997. Trabalhou com geofísica aplicada no Instituto de Pesquisas Tecnológicas do Estado de São Paulo de 1977 a 1997 . Atualmente é professor assistente doutor do Departamento de Geologia Aplicada do IGCE-UNESP onde atua desde 1997. Trabalha com métodos geofísicos aplicados à Hidrogeologia, Meio Ambiente, Geologia de Engenharia e Geotectônica.

Antonio Celso de Oliveira Braga. Bacharel em geologia pela Universidade Estadual Paulista-UNESP/Campus de Rio Claro (1978). Doutorado em Geociências pela Universidade Estadual Paulista-UNESP/Campus de Rio Claro (1997). Trabalhou no Instituto de Pesquisas Tecnológicas do Estado de São Paulo-IPT (1978 a 1995). Desde 1998 é Professor Doutor do Departamento de Geologia Aplicada-IGCE/UNESP - Rio Claro, atuando com geofísica aplicada à hidrogeologia e estudos ambientais, envolvendo os métodos geoelétricos. De 2002 a 2004, foi vice-coordenador do Programa de Pós-Graduação em Geociências - Área de Concentração em Geociências e Meio Ambiente-IGCE/UNESP. 